\title{
Antifungal itraconazole ameliorates experimental autoimmune encephalomyelitis through a novel mechanism of action
}

\author{
*Huifen Huang 1,B-D,F , *Xiaolin Tian 2,B,C,F, Xiao Peng ${ }^{1, C, F}$, Liangtong Huang ${ }^{1, D-F}$, Lerong Mei ${ }^{1, C, F}$, \\ Yanli Zhan ${ }^{1, D, F}$, Siying Chen ${ }^{1, B, F}$, Huihua Wu ${ }^{1, B, F}$, Guofang Wei ${ }^{1, D-F}$, Xueli Cai ${ }^{1, A, F}$ \\ ${ }^{1}$ Department of Neurology, Lishui Hospital, Zhejiang University School of Medicine, China \\ ${ }^{2}$ Department of Rehabitation Medicine, Second Hospital of Tianjin Medical University, China \\ A - research concept and design; $\mathrm{B}$ - collection and/or assembly of data; $\mathrm{C}$ - data analysis and interpretation; \\ $\mathrm{D}$ - writing the article; $\mathrm{E}$ - critical revision of the article; $\mathrm{F}$ - final approval of the article
}

Address for correspondence

Xueli Cai

E-mail: caixuelilsh@163.com

\section{Funding sources}

This research was supported by the Lishui public welfare technology application research selffunded project (project name: Study on the effect and mechanism of new AMPK activator against oligodendrocyte injury in multiple sclerosis; project No. 2019SJZ(30).

Conflict of interest None declared

* Huifen Huang and Xiaolin Tian contributed equally to this work.

Received on September 5, 2019

Reviewed on March 9, 2020

Accepted on April 24, 2020

Published online on May 27, 2020

\begin{abstract}
Background. Multiple sclerosis (MS) is an autoimmune disease characterized by a loss of myelin, limb disabilities and dysregulation of gene expression. Unfortunately, there still is no treatment to cure MS.

Objectives. To explore a novel way to treat MS using currently available antifungal drugs.

Material and methods. We built an experimental autoimmune encephalomyelitis (EAE) model to mimic MS and tested the effect of an antifungal drug - itraconazole - on EAE by comparing it with a phosphatebuffered saline (PBS) control group. We assessed the animal limb deficits with Weaver's scoring and used histology staining (including luxol fast blue (LFB) and hematoxylin \& eosin (H\&E) methods) to determine the demyelination in the spinal tissues. We also performed western blotting to quantify the expression changes of proteins related to endoplasmic reticulum (ER) stress response and apoptosis.
\end{abstract}

Results. The limb disabilities were greatly diminished and the demyelination in the spinal tissues of the EAE mice was mostly reduced following itraconazole treatment. The hyperactivation of the ER stress response and apoptosis pathway in EAE was also significantly diminished by the itraconazole treatment. In addition, the AMPK pathway was downregulated in EAE, its expression level bi-directionally affected the activity of the ER stress response, and its downregulation removed the beneficial effect of itraconazole.

Conclusions. Our study revealed a new method for treating MS using currently approved antifungal drugs.

Key words: apoptosis, EAE, itraconazole, ER stress, AMPK

Cite as

Huang H, Tian X, Peng X, et al. Antifungal itraconazole ameliorates experimental autoimmune encephalomyelitis through a novel mechanism of action. Adv Clin Exp Med. 2020;29(5):535-545. doi:10.17219/acem/121008

DOI

10.17219/acem/121008

Copyright

Copyright by Author(s)

This is an article distributed under the terms of the

Creative Commons Attribution 3.0 Unported (CC BY 3.0)

(https://creativecommons.org/licenses/by/3.0/) 


\section{Introduction}

Multiple sclerosis (MS) is an autoimmune disease which affects millions of people worldwide; in MS, the immune system improperly attacks its own tissues. ${ }^{1}$ Specifically, the immune system attacks the protective myelin - which envelopes the nerve fibres and facilitates fast neuronal impulses - and eventually affects the fast-neuronal conduction in myelinated fibers. Multiple sclerosis has 4 key pathological features ${ }^{2}$ :

- inflammation, which is considered to be the main trigger of the neuronal injuries;

- demyelination, the mark of MS;

- loss or damage of axons; and

- gliosis. $^{3}$

Experimental autoimmune encephalomyelitis (EAE), a primary animal model of autoimmune inflammatory diseases established over 80 years ago, ${ }^{4}$ is widely used in research on MS because it resembles MS in terms of inflammation, demyelination, apoptosis, and gliosis. ${ }^{5}$ Based on the gene expression in the EAE brain, ${ }^{6,7}$ some novel treatments involved in neuroprotection and immunosuppression have been developed. However, there is still no standard treatment that can fully cure MS due to the various symptoms of MS differing greatly from person to person and to the unclear molecular mechanism behind it. ${ }^{8}$ Therefore, it is valuable to further develop novel therapies in MS.

During the pathogenesis of EAE, several components of endoplasmic reticulum (ER) stress response have been found to be upregulated. ${ }^{9}$ The ER stress response, also known as the unfolded protein response, is critical for ameliorating the accumulation of unfolded or misfolded proteins and its prolonged activation can induce an inflammatory signal. ${ }^{10}$ The ER stress activates 3 branches of response to mitigate the stress, represented by activating transcription factor 6 (ATF6), inositol requiring enzyme 1 (IRE1) and PKR-like endoplasmic reticulum kinase (PERK) pathways. ${ }^{11}$ ATF6 helps increase the capacity of ER protein folding by being cleaved into transcription factor ATF6(N) and promoting the synthesis of folding-assisting proteins. ${ }^{12}$ PERK indirectly inhibits mRNA translation by phosphorylating itself and eIF2a (a ubiquitous translation initiation factor), thereby decreasing the load of proteins into the ER. Besides, PERK can also contribute to cell apoptosis by sequentially driving transcription factors ATF4 and CHOP (transcription factor C/EBP homologous protein) once the ER stress can no longer be handled. ${ }^{13}$ Phosphorylated IRE1 mitigates the ER response by degrading ER-binding mRNAs and enhancing transcriptional responses to increase the protein-folding capacity. ${ }^{14}$ Considering the hyperactivity of the ER stress response in EAE, it is worth attempting to treat MS by suppressing the ER stress responses.

Given that developing drugs from scratch is both timeconsuming and costly, screening currently available drugs is a reasonably promising idea. There is evidence which suggests that fungal infection can lead to MS and can cause an elevation of some biomarkers of MS, like interleukin 17 (IL-17) and chitotriosidase. ${ }^{15}$ Additionally, among several antifungal drugs, itraconazole has been shown to improve the condition of patients with psoriasis, which is another autoimmune disease, by binding to the fungal cytochrome p450 enzymes and inhibiting the production of ergosterol. ${ }^{16-18}$ Thus, we explored the novel effect of antifungal itraconazole on ameliorating MS-related symptoms and molecular changes using the EAE animal model and oligodendroglia OLN-93 cell line.

In this study, we successfully built an animal EAE model in which the animals exhibited severe limb disabilities and their spinal tissues lost myelin. We then tested the effect of itraconazole on the pathogenesis of EAE and found that the tissue lesions and animal motor deficit was dramatically diminished by itraconazole. Meanwhile, the pathways of the ER stress response and apoptosis were highly activated in EAE mice, and itraconazole application significantly restored the hyperactivation of these pathways. Furthermore, we confirmed the suppressive effect of itraconazole on the ER stress response and apoptosis by using an ER stress cell model induced by thapsigargin (Tg), a known cytotoxic inducer of ER stress. ${ }^{19}$ We additionally explored the novel mechanism of itraconazole mitigating EAE and found that the neuroprotective AMPK pathway was essential in this process, as knockdown of AMPK completely abolished the beneficial effect of itraconazole. Collectively, our results showed that this currently available antifungal drug might be used to help mitigate MS and provide a new direction in the future treatment of MS.

All the experimental procedures were approved by the ethics committee of Zhejiang University School of Medicine, China.

\section{Material and methods}

\section{EAE model}

The adult male C57BL/6J mice (8 weeks old) used in this study were ordered from Shanghai Laboratory Animal Center, China. The EAE model was built using antigen myelin oligodendrocyte glycoprotein (MOG) protein 35-55 (SCP0195; Sigma-Aldrich, St. Louis, USA) as previously described. ${ }^{20}$ Briefly, the mice were immunized by subcutaneous (s.c.) injection of $1.5 \mathrm{mg} / \mathrm{mL}$ of MOG 35-55 in complete Freund's adjuvant (CFA) on both the upper and lower back of each animal $(0.2 \mathrm{~mL}$ in total). Two hours later, each animal was intraperitoneally (i.p.) injected with $2 \mu \mathrm{g} / \mathrm{mL}$ of pertussis toxin (PTX, $0.1 \mathrm{~mL}$; Sigma-Aldrich). Forty-eight hours later, the mice received another i.p. injection of $2 \mu \mathrm{g} / \mathrm{mL}$ of PTX $(0.1 \mathrm{~mL})$. For the sham controls, phosphate-buffered saline (PBS) was delivered in each corresponding injection. To test the effect of the drug on the development of EAE, from 
the onset of immunization to the termination of the study, the animals were randomly grouped and received daily i.p. injection of $100 \mu \mathrm{L}$ of PBS, itraconazole $(10 \mathrm{mg} / \mathrm{kg})$, Compound C (also known as dorsomorphin, $20 \mathrm{mg} / \mathrm{kg}$ ), AICAR (5-aminoimidazole-4-carboxamide ribonucleotide, $500 \mathrm{mg} / \mathrm{kg}$ ), or combinations of the above. The EAE mice were examined daily from day 1 to 21 and scored for clinical symptoms. To test the effect of itraconazole on the acute ER stress response, another batch of mice received daily i.p. injections of $\mathrm{Tg}(0.4 \mathrm{mg} / \mathrm{kg})$, or Tg+itraconazole $(10 \mathrm{mg} / \mathrm{kg})$ without immunization. All drugs were purchased from Sigma-Aldrich. Twenty-one days after the first immunization or 15 days after the first Tg treatment, the mice were anesthetized with a mixture of ketamine $(80 \mathrm{mg} / \mathrm{kg})$ and xylazine $(10 \mathrm{mg} / \mathrm{kg})$. They were then perfused with saline and $4 \%$ paraformaldehyde and the spinal tissues were obtained for subsequent immunostaining or western blot analysis. The cytotoxicity of itraconazole was examined by staining the livers from the same animals.

\section{Downregulation or upregulation of AMPK in vivo}

The sequence of mus AMP-activated, alpha 1 catalytic subunit (AMPK $\alpha 1$, NM_001013367.3) was constructed into the pcDNA3.1 vector for overexpressing AMPK. The oligonucleotide sh-AMPK1 (GGAGAGCUAUUUGAUUAUA UU) and its control sh-NC (ACA CAA CGC GGA AUC UCG AAU), was synthesized and also constructed into pcDNA3.1 to knockdown AMPK1. All the constructs were then packaged into adeno-associated virus vectors (AAV) for in vivo experiments by GenePharma Co., Ltd. (Shanghai, China). To manipulate the level of AMPK, AAV-shAMPK or AAV-AMPK was transferred to some mice via tail vein injection of AAVs 10 days before the first immunization as previously described. ${ }^{21}$ The AAVs packed with a mock (sh-NC) or vector sequence were used as controls.

\section{Luxol fast blue and hematoxylin \& eosin staining}

The lumbar sections of the spinal cord $(15 \mu \mathrm{m})$ were prepared and stained using Luxol Fast Blue (LFB) Stain Kit (ab1506751; Abcam, Cambrdge, UK) according to the manual. The prepared liver sections were stained using the hematoxylin \& eosin (H\&E) method: the sections were first soaked in $0.4 \% \mathrm{HCl}$ (in $\mathrm{EtOH}$ ) for $15 \mathrm{~s}$, washed once, and then stained with eosin for $3 \mathrm{~min}$. The LFB and H\&E slides were then imaged to examine the pathological changes of tissues.

\section{Weaver score}

From day 0 to day 21 after immunization, the mice were scored daily to quantify their limb disabilities using Weaver's scoring method. ${ }^{22,23}$ Briefly, the total score is the sum of the response from the tail and all 4 limbs as follows: tail - 0 for normal, 1 for partially paralyzed and 2 for fully paralyzed; each limb - 0 for normal, 1 for weak gait, 2 for paresis, and 3 for fully paralyzed. Therefore, the total score ranges from $0-15$.

\section{ELISA assay}

First, the total proteins were obtained by centrifuging the fresh liver tissues obtained from the EAE mice. The concentration of the proteins of interest (AST, ALT and ALP) was measured using sandwich-type enzymelinked immunosorbent assay (ELISA) kits (Boster Bio, Pleasanton, USA). Briefly, the proteins of interest were sequentially captured with monoclonal antibody coated on a 96-well plate and linked with a biotin-conjugated detection antibody. Then, the biotin signal was amplified with avidin conjugated HRP and read using a microplate reader (Synergy2; BioTek, Winooski, USA) at $450 \mathrm{~nm}$. The concentration of the proteins was calculated using the standard curve method.

\section{Western blot}

The spinal cord segments or OLD cells were first treated with radioimmunoprecipitation assay (RIPA) buffer to lysate the cells (Thermo Fisher Scientific, Waltham, USA) following the manufacturer's instructions. The proteins were then separated using gel electrophoresis, followed by incubation with primary and secondary antibodies conjugated with horseradish peroxidase (HRP) and visualization. The protein level was quantified using ImageJ software (National Institutes of Health, Bethesda, USA), normalized to the value of internal control, GAPDH or Actin- $\beta$, and expressed as the fold of the control. The primary antibodies were purchased from Thermo Fisher Scientific (p-PERK, \#MA5-15033; ATF4, \#PA586112; p-IRE1 $\alpha$, \#PA1-16927; GAPDH, \#39-8600; Bax, \#MA5-14003; $\beta$-actin, \#MA5-11869; AMPK- $\alpha 1$, \#MA515815; and ATF6, \# MA1-25358), Cell Signaling Technology (Danvers, USA; CHOP, \#2895; p-eIF2 $\alpha$, \#9721; and p-AMPK $\alpha$, \#2531), and Abcam (caspase-3, ab13847; Bcl-2, ab182858; and p53, ab26)

\section{Cell culture and Cell Counting Kit- 8 assay}

Due to the critical role of oligodendrocytes (OLs) in producing and maintaining the myelin around axons, ${ }^{24}$ for cellular study we used the OLN-93 cell line (Otwo Biotech, Shenzen, China), which is derived from rat brain cultures. ${ }^{25}$ The OLN cells were cultured in Gibco Dulbecco's modified Eagle's medium (DMEM) basal media containing a high level of glucose (Thermo Fisher Scientific) at $37^{\circ} \mathrm{C}$. The dosage-dependent cytotoxicity of Tg and itraconazole was determined with a Cell Counting Kit-8 (CCK-8; Dojindo Laboratories, Kumamoto, Japan; CK04). 
Briefly, the OLN cells were precultured in 96-well plates for $12 \mathrm{~h}$ (5,000 cells/well) and then treated with various concentrations $(0,1,5,10,20$, and $40 \mathrm{nM})$ of Tg and itraconazole for $24 \mathrm{~h}$. Next, $10 \mu \mathrm{L}$ of CCK- 8 solution was added, and the cells were cultured for another $3 \mathrm{~h}$. Finally, the cellular optical density (OD) was measured at $450 \mathrm{~nm}$, and the percentage of cell viability was calculated as the ratio of samples to controls. To test the effect of itraconazole on the activity of the ER stress response evoked by $\mathrm{Tg}, \mathrm{Tg}$ (20 $\mathrm{nM}$ ) or a combination of Tg and itraconazole (5 nM) was added and the cells were cultured for another $48 \mathrm{~h}$ before protein analysis using western blotting.

\section{Flow cytometry}

For the apoptosis test, the OLN cells were treated with PBS, Tg (20 nM), or Tg+itraconazole (20 nM+5 nM) for 48 h. Then, the OLN cells were washed twice with PBS, digested using EDTA-free trypsin, and treated using fluorescent annexin V conjugates (Thermo Fisher Scientific) according to the instructions. The percentage of cell apoptosis was quantified using a flow cytometer (Beckman Coulter Inc., Brea, USA) with an excitation wavelength of $488 \mathrm{~nm}$.

\section{Statistical analysis}

The sample size in each drug/virus treatment group was 6 animals. In total, there were 19 groups and 114 mice (Fig. 1-6; all animals used for behavior assessment were also used for tissue staining and western blotting). An example band from 1 or 2 animals was shown in western blotting. For the cellular study (testing drug toxicity using CCK-8, examining apoptosis using flow cytometry and measuring the activity of the ER stress response or apoptosis induced by $\mathrm{Tg}$ ), experiments were repeated 3 times for quantification. The data is expressed as means \pm standard deviation (SD). The confidence level was set at 0.05 . Statistical differences were analyzed using Student's t-test between 2 groups or one-way analysis of variance (ANOVA) followed by Dunnett's test among multiple groups, using Prism 6 (GraphPad Software Inc., San Diego, USA).

\section{Results}

\section{Itraconazole functionally mitigated the tissue damage and limb disabilities in EAE mice}

We first built the EAE animal model by immunizing the mice with MOG and found that the tissue damage (indicated by infiltration and demyelination) in the lumbar spinal cord was severe (Fig. 1A). Meanwhile, the mice exhibited significant limb deficits as shown by an increase in the clinical Weaver scores (Fig. 1B). These behavioral and histological changes suggested that the EAE model had been successfully built. Next, we tested whether the application of the antifungal itraconazole could rescue these phenotypes. We found that the demyelination in EAE was largely restored (Fig. 1A), and the animal limb disabilities were significantly lessened (Fig. 1B). To make sure that the chosen dosage of itraconazole did not cause any cytotoxicity that was not observed in the spinal tissues, we performed H\&E staining using the livers obtained from the same EAE animals; no obvious liver pathogenesis was observed (Fig. 1C). Meanwhile, the level of liver failure markers - AST, ALT and ALP - was also not significantly different between the EAE+itraconazole group and the EAE+PBS group (Fig. 1D). Together, this data suggested that the nontoxic dosage of antifungal itraconazole very effectively diminished the tissue damage and limb disabilities in EAE.

\section{Itraconazole diminished the overactivation of the ER stress response and cell apoptosis in EAE}

We next explored the novel mechanism of itraconazole in regulating the symptoms of EAE. We measured the level of ER stress response-related proteins and found that the levels of cleaved ATF6, phosphorylated-PERK (p-PERK), p-eIF-2 $\alpha$, ATF4, CHOP, and p-IRE $\alpha$ were all much higher in EAE (Fig. 2A,B). These changes indicated that all 3 branches of ER stress response were concurrently dysregulated in EAE and the endogenous mechanisms to mitigate the stress were disrupted. Importantly, the application of itraconazole largely restored the overactivity of the proteins involved in ER stress response (Fig. 2A,B). As cell apoptosis is also one of the main symptoms in EAE, we also examined the expression change of apoptotic indicators. We found that apoptotic activators, including caspase-3, Bax and TP53, exhibited high expression levels while there were lower levels of the apoptotic inhibitor $\mathrm{Bcl}-2$ in EAE. Accordingly, these changes were also significantly reduced by itraconazole (Fig. 2C,D). Interestingly, the level of p-AMPK, which is key in cellular energy metabolism, was also decreased in EAE and improved by administering itraconazole (Fig. 2A,B). Together, these results indicate that itraconazole effectively restored the dysregulated gene expression network involved in the EAE-related ER stress response and cell apoptosis.

\section{Itraconazole mitigated the cell apoptosis and hyperactivity of ER stress response induced by Tg in OLN cells}

To further confirm the mitigating effect of itraconazole on the ER stress response, which might be important in the development of EAE, we acutely induced an ER stress response in OLN cells using Tg. We first tested the dosage effect of $\mathrm{Tg}$ and itraconazole on cell survival 
A
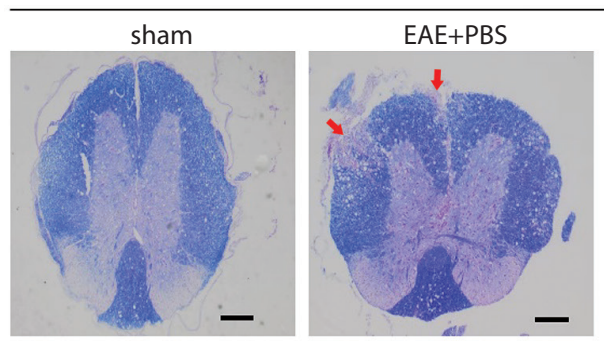

Luxol Fast Blue staining (spinal cord)
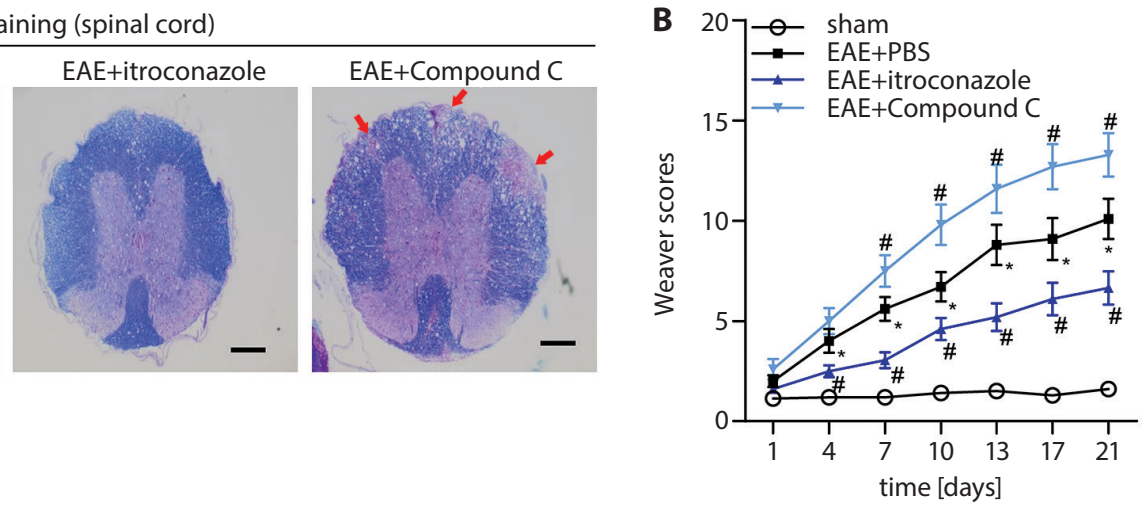

C

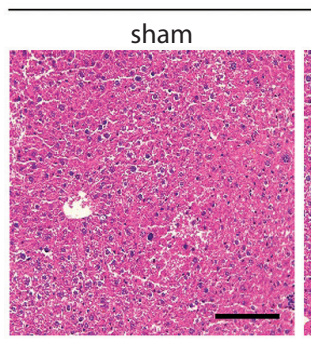

H\&E staining (liver cytotoxity)
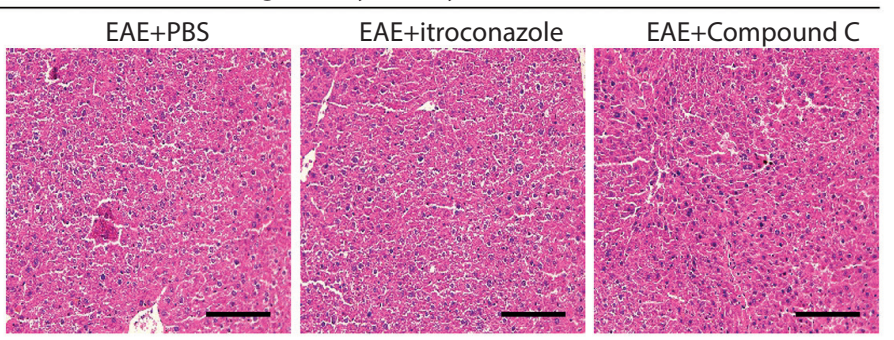

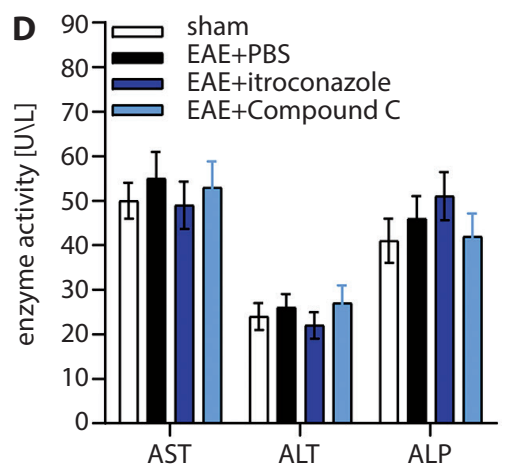

Fig. 1. Itraconazole effectively diminished the tissue damage and limb disabilities in EAE. A. Representative LFB-stained transverse sections of spinal cord showing the demyelination (pallor and vacuolation indicated by arrow) induced by EAE. The demyelination was corrected and exacerbated by itraconazole and Compound C (an AMPK pathway inhibitor) treatment, respectively. Scale bar, $200 \mu$ m. B. Itraconazole dramatically alleviated the limb disabilities, as shown by lowered Weaver scores, while Compound C exacerbated the disabilities. C. Representative H\&E-stained liver sections showing that the dosages of both itraconazole and Compound C used in the study were not cytotoxic to the liver. Scale bar, 100 Mm. D. The levels of liver failure markers, such as AST, ALT and ALP, was not significantly affected by itraconazole or Compound C treatment, as assessed using ELISA. One-way ANOVA followed by Dunnett's test

${ }^{*} p<0.01$ vs the sham group; \# $p<0.01$ vs the EAE+PBS group.

and found that they displayed significant cytotoxicity at 20-40 nM but not at a lower dosage (5 nM; Fig. 3A). We next examined whether the nontoxic dosage of itraconazole $(5 \mathrm{nM})$ could reverse the cell apoptosis induced by $20 \mathrm{nM}$ of $\mathrm{Tg}$ and found that the percentage of cell death decreased from $\sim 20 \%$ in the $\mathrm{Tg}$ group to $\sim 7.5 \%$ in the $\mathrm{Tg}+\mathrm{itraconazole}$ group (Fig. 3B). At the protein level, $20 \mathrm{nM}$ of $\mathrm{Tg}$ increased the expression of apoptotic activators (caspase-3, Bax and TP5) and decreased the expression of inhibitors (Bcl-2) in OLN cells (Fig. 3C,D). In contrast, these changes were dramatically diminished by itraconazole administration (Fig. 3C,D). We further confirmed the potential anti-apoptotic effect of itraconazole using tissues obtained from animals pretreated with PBS, Tg or Tg+itraconazole. Consistently, we found the dysregulation of apoptotic mediators significantly ameliorated by itraconazole (Fig. 3E,F). We further examined whether itraconazole could reverse the overactivation of the ER stress response in the same tissues and found that the upregulation of ER stress response mediators caused by Tg was significantly decreased by itraconazole (Fig. 4). Overall, these results further demonstrated that itraconazole could suppress the activity of cell apoptosis and the ER stress response.

\section{AMPK was a novel mediator of itraconazole mitigating the hyperactivity of the ER stress response and apoptosis}

We next explored the novel mediator of itraconazole restoring the dysregulated gene network relevant to the apoptosis and ER stress response in EAE. We noticed that the level of p-AMPK dropped significantly in the spinal cords of EAE mice (Fig. 2A,B). Previous studies reported that the activity of AMPK was reduced in the brain from EAE disease and the genetic ablation of AMPK caused severe clinical impairments in EAE animals. ${ }^{26-28}$ We first validated the functional role of the AMPK pathway in the development of EAE. Consistent with the effect of genetic ablation of AMPK, suppressing the AMPK pathway with Compound $\mathrm{C}$ significantly exacerbated the demyelination in the spinal cord and further impacted the clinical score compared with the EAE+PBS group (Fig. 1A,B), although the same dosage was not cytotoxic for the liver (Fig. 1C,D). Besides, the level of AMPK pathway also affected the activity of the ER stress response, as AICAR - an activator of AMP-activated protein kinase (AMPK) - statistically diminished the hyperactivation of ER-stress-related proteins 
A

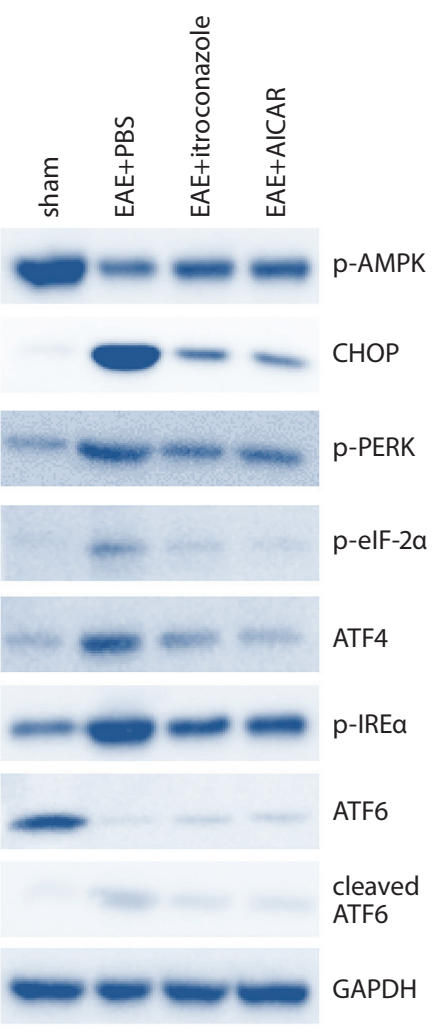

C

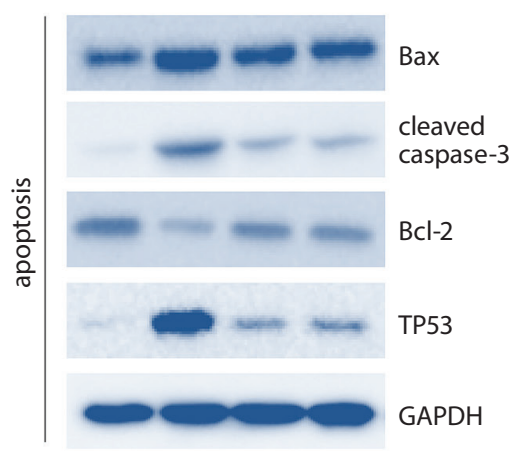

B
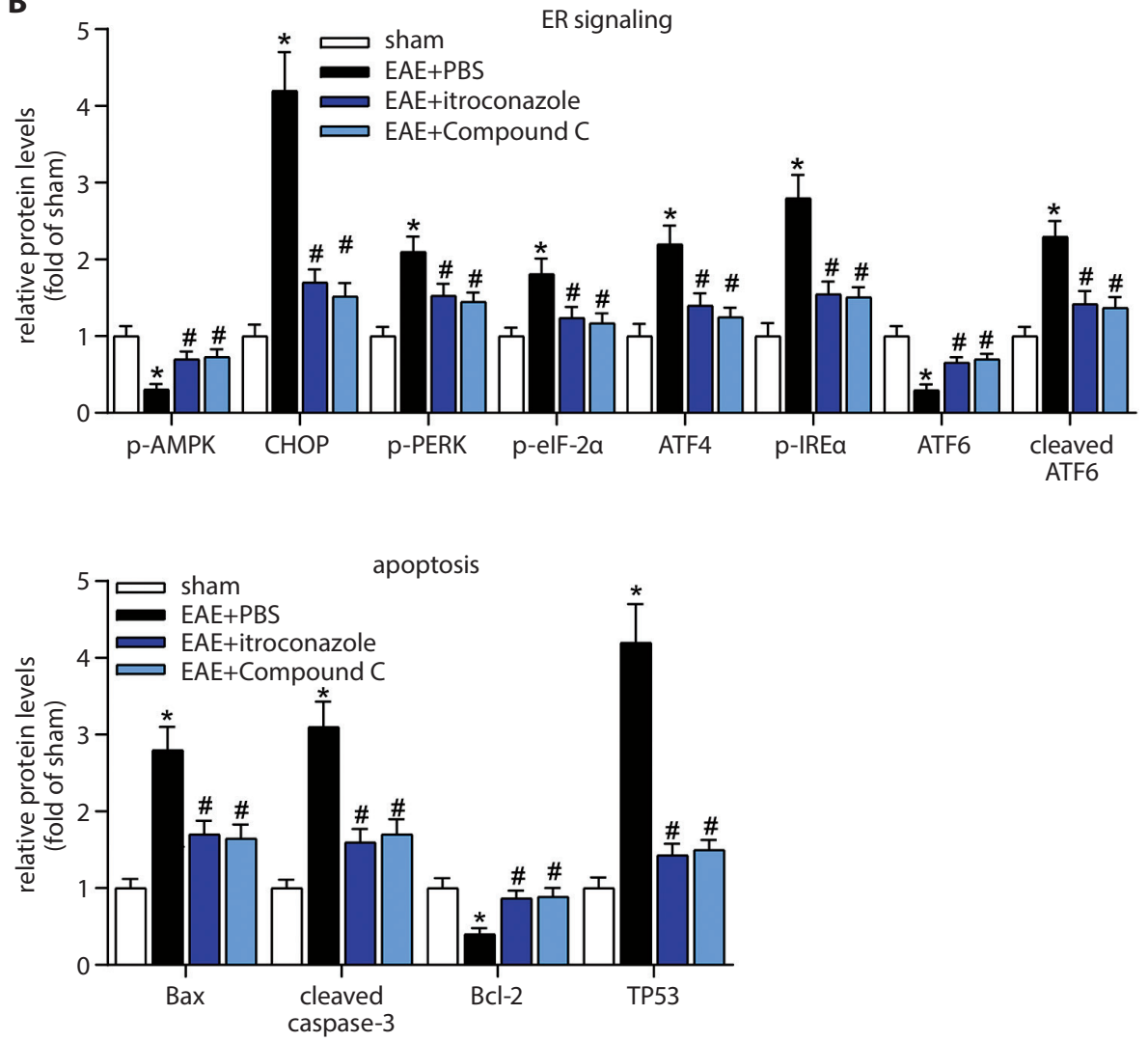

Fig. 2. Itraconazole mitigated the hyperactivity of the ER stress response and cell apoptosis in EAE. A. The levels of ER stress response mediators, including ATF6, PERK and IRE, were upregulated, while the level of p-AMPK was downregulated in spinal tissues of EAE mice. Both itraconazole and AICAR (activator of AMPK pathway) restored the changes. B. Quantitative analysis of the proteins' levels in (A). C and D. Expression change of apoptotic mediators was significantly improved by itraconazole and AICAR. One-way ANOVA followed by Dunnett's test

* $p<0.01$ vs the sham group; \# $p<0.01$ vs the EAE+PBS group.

(Fig. 2A-D). Meanwhile, similar effects were obtained in the mice treated with the acute ER stress inducer $\mathrm{Tg}$ (Fig. 4). As Compound C is also a BMP pathway inhibitor, we further determined the role of AMPK in EAE by delivering AAV transgenes to the mice to specifically knockdown (AAV-sh-AMPK) or overexpress AMPK (AAV-AMPK) before EAE. Consistent with the pharmacological results, the knockdown of AMPK further enhanced the activity of ER stress response and apoptosis, while overexpression of AMPK largely reduced those responses (Fig. 5A-D). Taken together, these complementary manipulations demonstrated that the AMPK pathway could bi-directionally regulate the development of EAE at the animal motor, tissue and molecular levels.
In our study, we found that the downregulation of p-AMPK was reversed by itraconazole in EAE (Fig. 2A,B). Therefore, we hypothesized that an elevation of $\mathrm{p}$-AMPK might be required for the beneficial effect mediated by itraconazole. As expected, we found that the downregulation of AMPK further enhanced the hyperactivity of the ER stress response, while itraconazole decreased the response in the spinal tissues of Tg-treated animals (Fig. 6A,B). Importantly, the beneficial effect of itraconazole was abolished by AMPK knockdown (Fig. 6A,B). Therefore, AMPK might be a key downstream target of itraconazole in order to mitigate the hyperactivity of apoptosis and the ER stress response in EAE. 
A

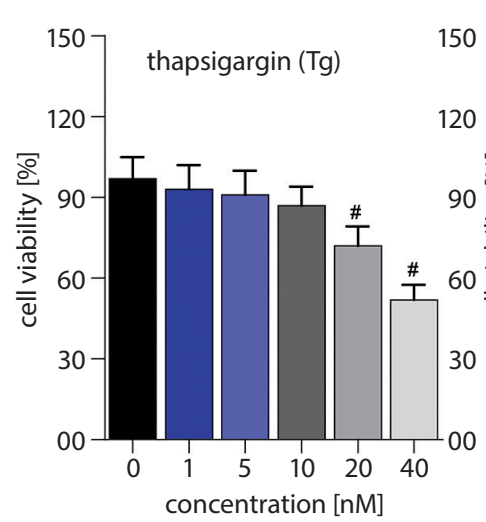

C ONL cells
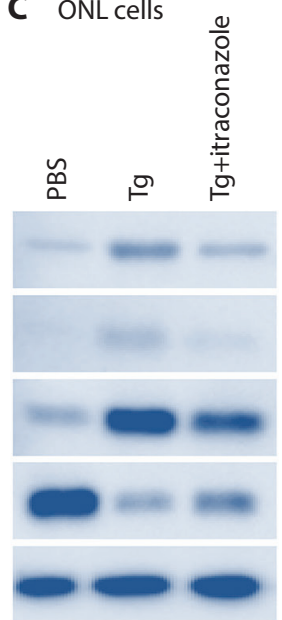

Bax

cleaved

caspase-3

TP53

Bcl-2

actin- $\beta$
B
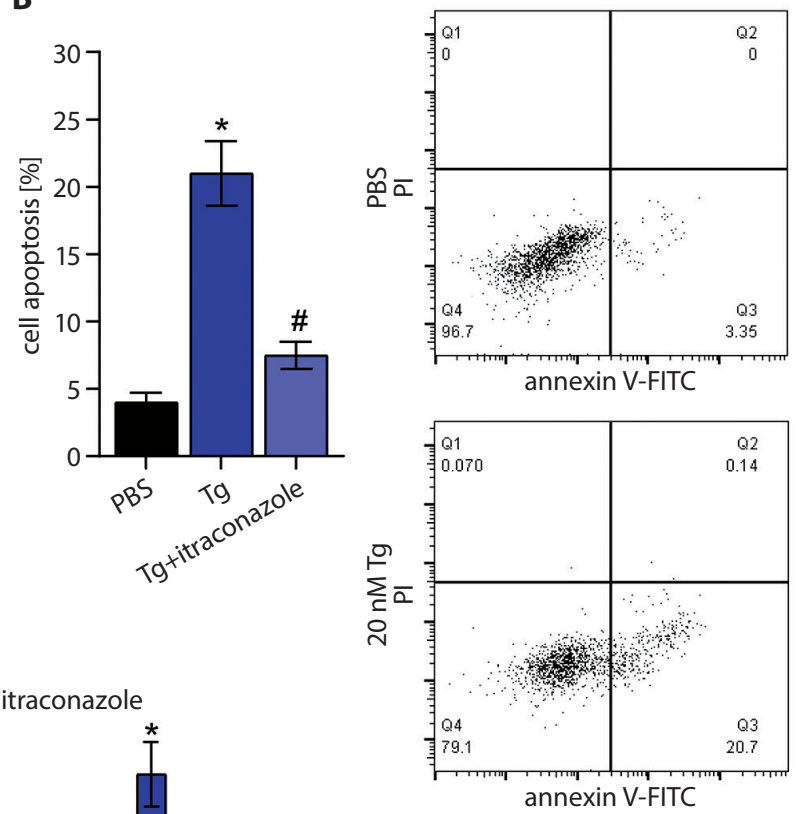

D ONL cells

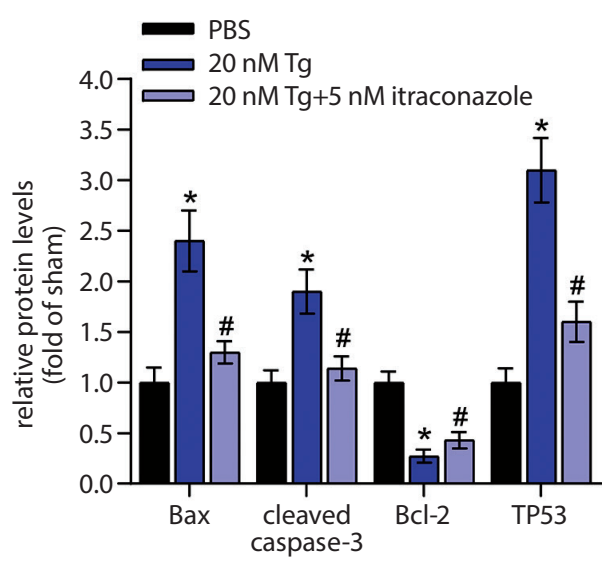

F animal tissues

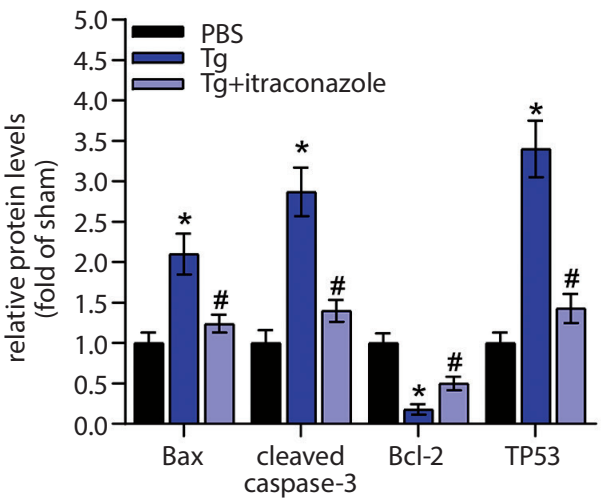

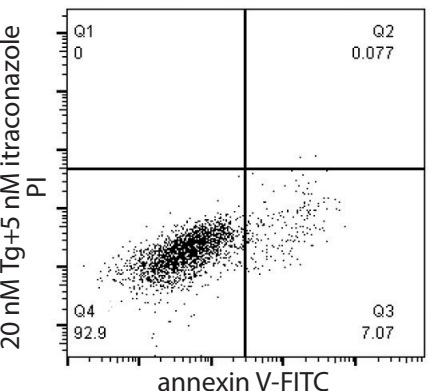

E animal tissues

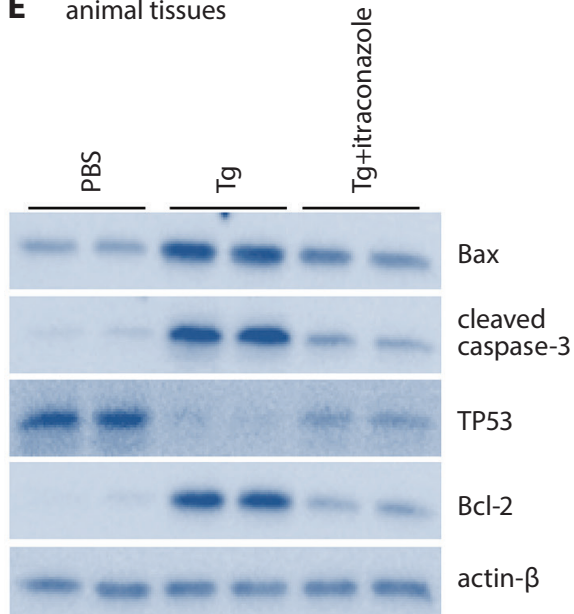

Fig. 3. Itraconazole mitigated the cell apoptosis caused by ER stress inducer Tg at the cellular and protein levels. A. Dosage effects of Tg and itraconazole on the cell viability of OLN cells as assessed using a CCK-8 assay. The cell viability gradually decreased with increasing concentration of Tg and itraconazole. One-way ANOVA followed by Dunnett's test; \# $\mathrm{p}<0.05 \mathrm{vs} 0 \mathrm{mM}$. B. The application of itraconazole significantly decreased the percentage of OLN cell apoptosis evoked by Tg determined using flow cytometer. The altered levels of apoptotic mediators induced by Tg in the OLN cells (C and D) or animal tissues (E and F) was significantly reversed by itraconazole. One-way ANOVA followed by Dunnett's test

${ }^{*} p<0.01$ vs the PBS group; $\#<0.01$ vs the Tg group.

\section{Discussion}

Multiple sclerosis is an autoimmune disease in which the immune system destroys the insulation covering of nerves, thus affecting the fast conduction of neural impulse in myelinated fibers. Along with the development, MS is sequentially characterized by inflammation, demyelination, axonal loss, gliosis, and limb motor deficit, ${ }^{3}$ 
A

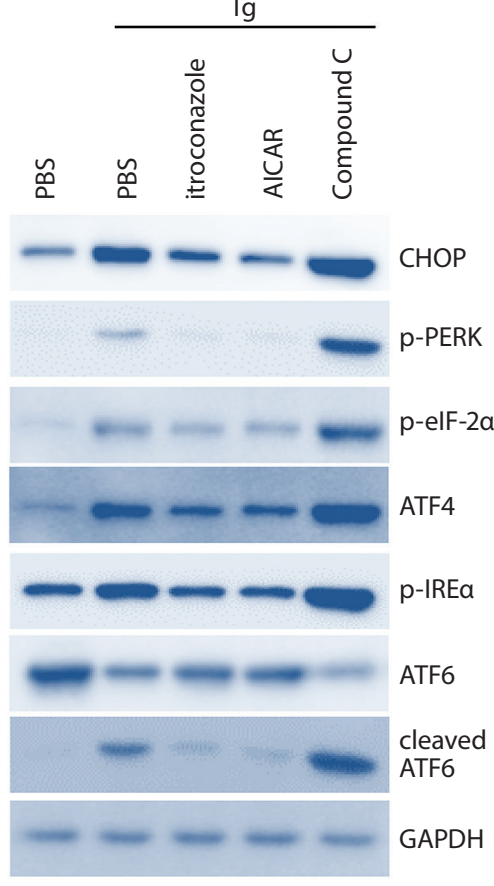

B

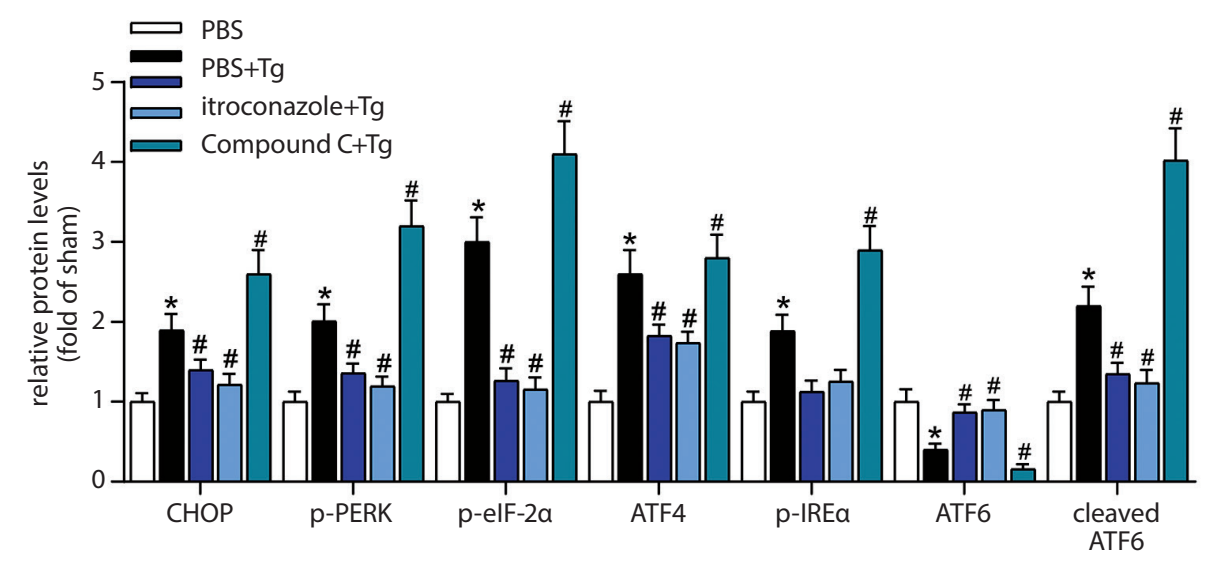

Fig. 4. Itraconazole mitigated the overactivity of the ER stress response evoked by Tg in spinal tissues. A and B. Itraconazole and AMPK signal activator AICAR diminished the overactivation of the ER stress pathway, while AMPK signal inhibitor Compound C further enhanced the overactivation. One-way ANOVA followed by Dunnett's test

${ }^{*} p<0.01$ vs the PBS group; \# $p<0.01$ vs the PBS+Tg group.

while the inflammatory and apoptotic responses at the cellular level gradually increased in MS patients. ${ }^{29}$ In our EAE model, we successfully replicated these symptoms: demyelination in the tissue of the lumbar spinal cord (Fig. 1A), severe limb disabilities (Fig. 1B), and overactivity of ER stress and apoptotic responses (Fig. 2). According to the development of MS, some EAE therapies have focused on suppressing the immune response and protecting the loss of nerves. For example, mitoxantrone was used for general immunosuppression ${ }^{30}$ and flecainide (Na channel blocker) was used for axonal protection. ${ }^{31}$ In our case, we significantly diminished neural damage and animal limb deficit using the antifungal itraconazole (Fig. 1A,B). A previous study found that fungal infection can lead to MS, and that some fungal-infection-related molecules are biomarkers of MS, laying the foundation to treat MS using antifungal drugs. ${ }^{15}$ Although our study opens a new window to treat MS using currently approved antifungal drugs, the translation from animal study to clinical trials needs further assessment, as the EAE model cannot completely mimic the symptoms of MS, and the biological process of MS and the effective dosage of itraconazole might greatly differ between animals and humans.

Due to the complexity of causes and symptoms in MS, the underlying biological mechanism is still very unclear. Studies using animal EAE model have uncovered that several components of the ER stress response are dysregulated at the cellular level. The ER stress response is a bifunctional process, determining the fidelity of protein folding in healthy cells and promoting cellular apoptosis once the prolonged ER stress cannot be resolved through 3 molecular branches. ${ }^{32}$ In our study, the level of mediators of all 3 branches in the ER stress response was changed in the spinal tissues of EAE mice (Fig. 2A). The increase of cleaved ATF6 and phosphorylation of IRE $\alpha$ indicated that the injured cells had tried to increase the protein-folding capacity in order to mitigate the ER stress in EAE. The increase of phosphorylated PERK, eIF- $2 \alpha$ and IRE $\alpha$ reflected that the cells attempted to reduce the flux of protein into ER to alleviate the ER stress, as these proteins can inhibit the mRNA translation and degrade ER-bound mRNAs. Meanwhile, the increase of transcription factor ATF4 and CHOP indicated that the self-death procedure was initiated as these factors can promote the transcription of apoptotic genes. Combined with the fact that the ER stress response has also been found to be an important feature in many other human diseases, ${ }^{33-36}$ our data suggest that the ER stress response might play important roles in endogenous self-repair and apoptosis in MS. Importantly, the antifungal itraconazole effectively inhibited the overactivation of ER stress response evoked by EAE (Fig. 2A,B) or Tg (Fig. 4A,B). Furthermore, the upregulation of apoptotic pathways in EAE or Tg treatment was also largely reversed by itraconazole (Fig. 2C,D and 3B-F). These results indicate that itraconazole can effectively restore the dysregulated molecular network caused by EAE, laying the molecular basis for alleviating EAE. 
A

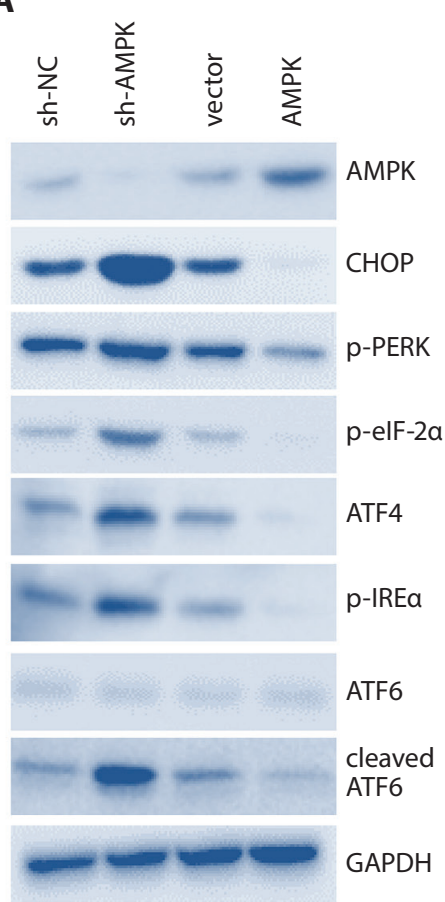

C
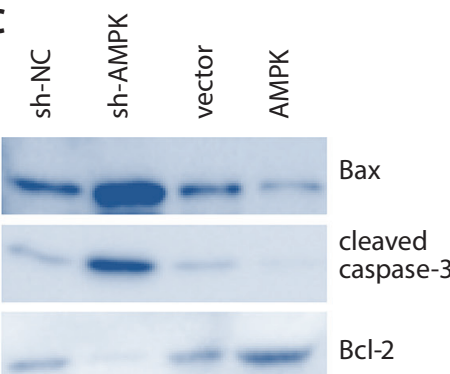

fP553

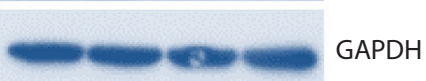

B

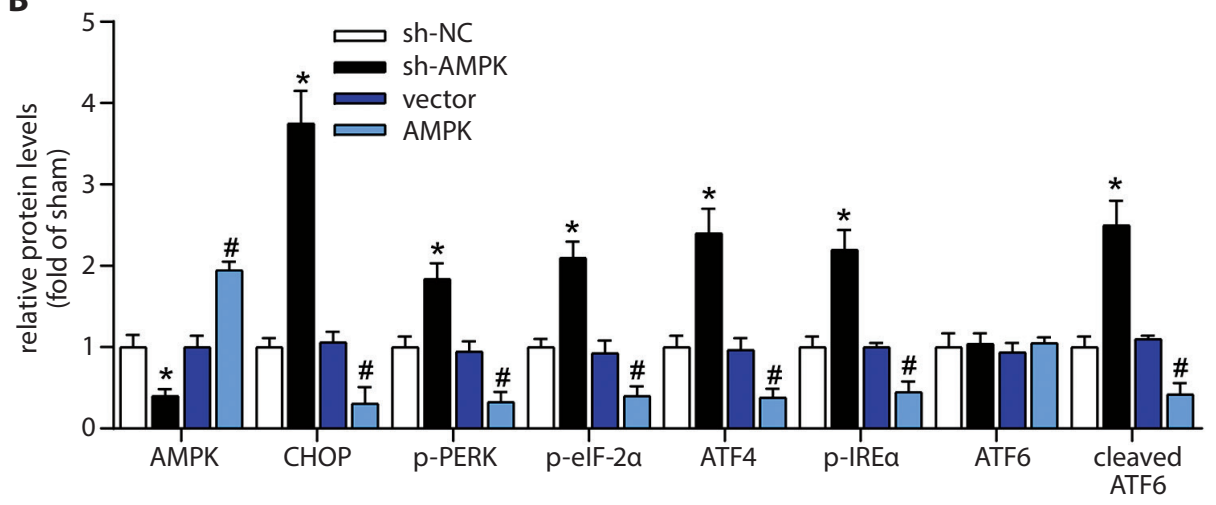

D

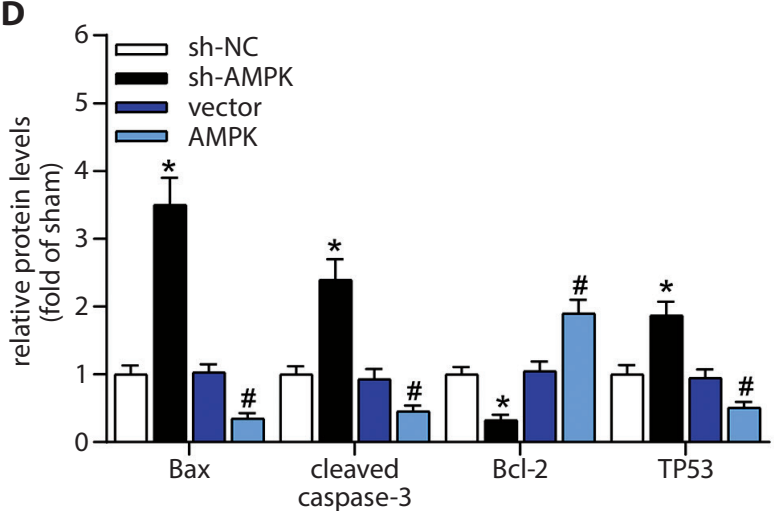

Fig. 5. The level of AMPK bi-directionally affected the activity of the ER stress response and apoptosis in spinal tissues of EAE. A and B. Knockdown or overexpression of AMPK by AAV delivery increased or decreased the activity of the ER stress response pathway, respectively. C and D. Knockdown or overexpression of AMPK increased or decreased the level of apoptotic biomarkers, respectively. Student's t-test

${ }^{*} p<0.01$ vs the sh-NC group; $\# p<0.01$ vs the vector group.

We found that one potential mediator of itraconazole ameliorating EAE was AMPK. The AMPK pathway is responsible for maintaining energy homeostasis by promoting the uptake of glucose and fatty acids and is also one of the central regulators of growth. ${ }^{37-39}$ Additionally, AMPK was relevant to the development of EAE, as its activity was reduced in the brain of EAE animals and the genetic ablation of AMPK exacerbated the clinical impairments in EAE animals. ${ }^{26-28,40}$ Consistent with these studies, our data showed that the phosphorylated level of AMPK largely decreased in EAE animals (Fig. 2A), further strengthening the correlation between the dysregulation of the AMPK pathway and EAE development. In addition, our study first showed that pharmacologically enhancing the AMPK pathway by AICAR significantly restored the hyperactivity of the ER stress response and apoptosis induced by EAE or Tg (Fig. 2,4), consistent with a previous finding that AICAR treatment protected inflammation-induced myelin loss and axonal abnormalities in EAE mice. ${ }^{41}$ Besides, specific downregulation or overexpression of AMPK with AAVs bi-directionally altered the endogenous activity of the ER stress response and cellular apoptosis in EAE animal tissues (Fig. 5). Therefore, our complimentary experiments demonstrated that the level of AMPK was critical for the molecular changes associated with EAE. Furthermore, the downregulation of endogenous AMPK abolished the mitigating effect of itraconazole on the acute ER stress response (Fig. 6). This result should not be simply 
A

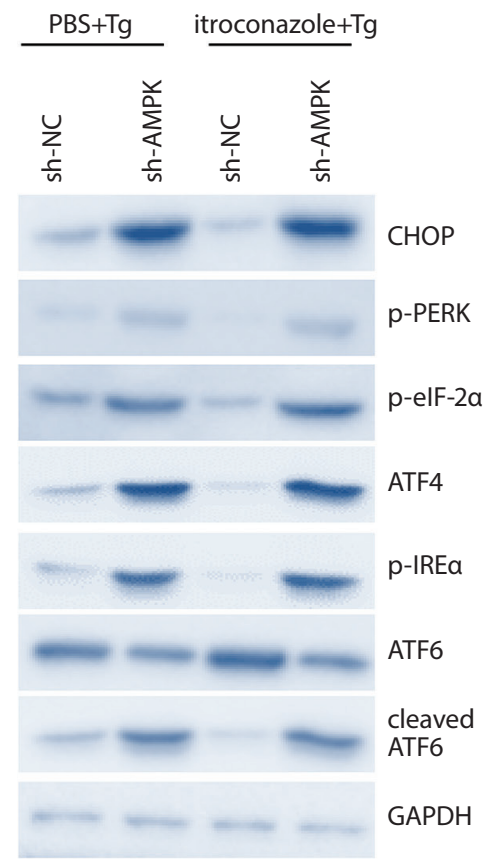

B

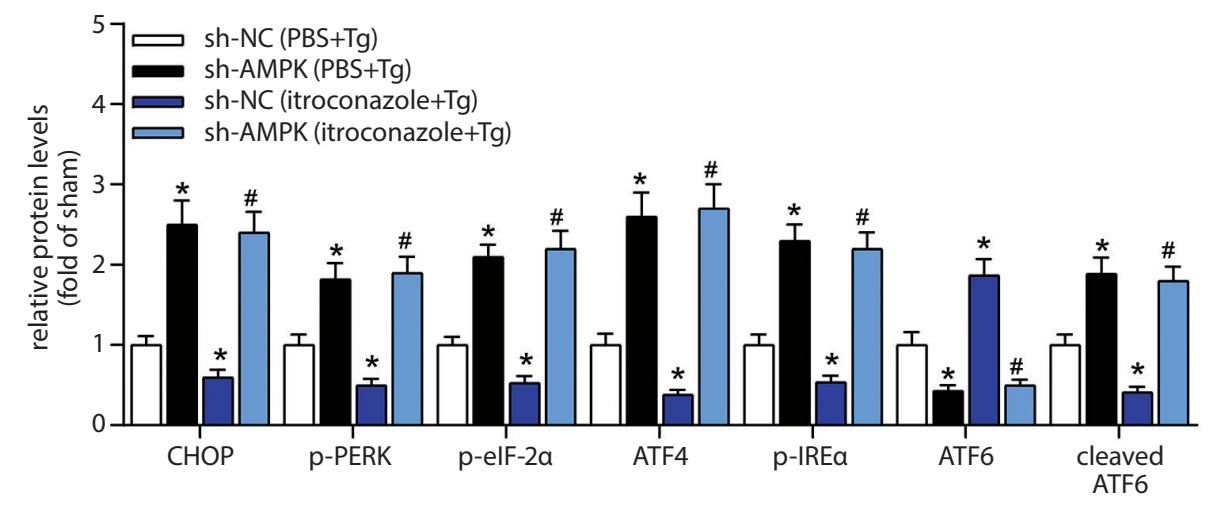

Fig. 6. Downregulation of AMPK removed the suppression effect of itraconazole on the overactivation of the ER stress response evoked by Tg in spinal tissues. A and B. The knockdown of AMPK further increased the hyperactivity of the ER stress response pathway induced by Tg and completely blocked the beneficial effect mediated by itraconazole. One-way ANOVA followed by Dunnett's test

${ }^{*} p<0.01$ vs the sh-NC (PBS+Tg) group; \# $p<0.01$ vs the sh-NC (itraconazole+Tg) group.

explained away by the fact that the beneficial effect of itraconazole was masked by the knockdown effect of AMPK. If that is the case, the levels of the proteins should be lower in the sh-AMPK (itraconazole+Tg) group than in the shAMPK (PBS+Tg) group, since AMPK and itraconazole independently regulated the response. This data indicates that the AMPK pathway might be an essential downstream mediator of itraconazole which mitigates ER stress and suggests that this pathway might be a good target for alleviating MS. It is crucial to explore how itraconazole regulates the AMPK pathway in order to find ways to enhance the therapeutic effect of itraconazole in MS.

\section{Conclusion}

We showed that the antifungal itraconazole effectively ameliorated the EAE symptoms at the behavioral, tissue, cellular, and molecular level. Specifically, the limb disabilities were significantly lessened as demonstrated by decreased clinical score. The demyelination in the spinal cord of EAE mice and the cell apoptosis percentage induced by Tg was largely decreased. Meanwhile, the hyperactivation of related molecular pathways, including ER stress response and cell apoptosis, was mostly reset to the basal level after itraconazole application. Furthermore, we verified that the AMPK pathway is involved in regulating EAE and is a novel target of itraconazole, since the beneficial effect of itraconazole was removed by knockdown of AMPK.
Together, these results demonstrated that the antifungal itraconazole mitigated EAE and showed a new direction to find ways to treat MS using the available antifungal drug.

\section{ORCID iDs}

Huifen Huang (10) https://orcid.org/0000-0003-2132-3733 Xiaolin Tian (1) https://orcid.org/0000-0003-2397-8151 Xiao Peng (10 https://orcid.org/0000-0003-2597-9473 Liangtong Huang (1) https://orcid.org/0000-0002-3105-8606 Lerong Mei (1) https://orcid.org/0000-0002-7227-8787 Yanli Zhan (1) https://orcid.org/0000-0002-8382-2880 Siying Chen (1) https://orcid.org/0000-0002-9563-2726 Huihua Wu (1) https://orcid.org/0000-0002-6345-2325 Guofang Wei (1) https://orcid.org/0000-0002-0512-1181 Xueli Cai (1) https://orcid.org/0000-0003-1670-3539

\section{References}

1. Browne P, Chandraratna D, Angood C, et al. Atlas of Multiple Sclerosis 2013: A growing global problem with widespread inequity. Neurology. 2014;83(11):1022-1024.

2. Goverman J. Autoimmune T cell responses in the central nervous system. Nat Rev Immunol. 2009;9(6):393-407.

3. Constantinescu CS, Farooqi N, O'Brien K, Gran B. Experimental autoimmune encephalomyelitis (EAE) as a model for multiple sclerosis (MS). Br J Pharmacol. 2011;164(4):1079-1106.

4. Rivers TM, Sprunt DH, Berry GP. Observations on attempts to produce acute disseminated encephalomyelitis in monkeys. J Exp Med. 1933;58(1):39-53.

5. Farooqi N, Gran B, Constantinescu CS. Are current disease-modifying therapeutics in multiple sclerosis justified on the basis of studies in experimental autoimmune encephalomyelitis? J Neurochem. 2010;115(4):829-844.

6. Han MH, Hwang SI, Roy DB, et al. Proteomic analysis of active multiple sclerosis lesions reveals therapeutic targets. Nature. 2008;451(7182): 1076-1081. 
7. Steinman L. A molecular trio in relapse and remission in multiple sclerosis. Nat Rev Immunol. 2009;9(6):440-447.

8. English C, Aloi JJ. New FDA-approved disease-modifying therapies for multiple sclerosis. Clin Ther. 2015;37(4):691-715.

9. Kamarehei M, Kabudanian Ardestani S, Firouzi M, et al. Increased expression of endoplasmic reticulum stress-related caspase-12 and CHOP in the hippocampus of EAE mice. Brain Res Bull. 2019;147:174-182.

10. Schroder M. Endoplasmic reticulum stress responses. Cell Mol Life Sci. 2008;65(6):862-894

11. Walter $P$, Ron $D$. The unfolded protein response: From stress pathway to homeostatic regulation. Science. 2011;334(6059):1081-1086.

12. Haze K, Yoshida H, Yanagi H, Yura T, Mori K. Mammalian transcription factor ATF6 is synthesized as a transmembrane protein and activated by proteolysis in response to endoplasmic reticulum stress. Mol Biol Cell. 1999;10(11):3787-3799.

13. Marciniak SJ, Yun CY, Oyadomari S, et al. CHOP induces death by promoting protein synthesis and oxidation in the stressed endoplasmic reticulum. Genes Dev. 2004;18(24):3066-3077.

14. Reimold AM, Iwakoshi NN, Manis J, et al. Plasma cell differentiation requires the transcription factor XBP-1. Nature. 2001;412(6844): 300-307.

15. Benito-Leon J, Laurence M. The role of fungi in the etiology of multiple sclerosis. Front Neurol. 2017;8:535.

16. Faergemann J. Treatment of sebopsoriasis with itraconazole. Mykosen. 1985;28(12):612-618.

17. Boehncke WH, Schon MP. Psoriasis. Lancet. 2015;386(9997):983-994.

18. Ahmad N, Mukhtar H. Cytochrome p450: A target for drug development for skin diseases. J Invest Dermatol. 2004;123(3):417-425.

19. Denmeade SR, Isaacs JT. The SERCA pump as a therapeutic target: Making a "smart bomb" for prostate cancer. Cancer Biol Ther. 2005; 4(1):14-22.

20. Berard JL, Wolak K, Fournier S, David S. Characterization of relapsingremitting and chronic forms of experimental autoimmune encephalomyelitis in C57BL/6 mice. Glia. 2010;58(4):434-445.

21. Grames MS, Jackson KL, Dayton RD, Stanford JA, Klein RL. Methods and tips for intravenous administration of adeno-associated virus to rats and evaluation of central nervous system transduction. J Vis Exp. 2017(126):55994. doi:10.3791/55994

22. Weaver A, Goncalves da Silva A, Nuttall RK, et al. An elevated matrix metalloproteinase (MMP) in an animal model of multiple sclerosis is protective by affecting Th1/Th2 polarization. FASEB J. 2005;19(12): 1668-1670.

23. Dasilva AG, Yong VW. Expression and regulation of matrix metalloproteinase-12 in experimental autoimmune encephalomyelitis and by bone marrow derived macrophages in vitro. J Neuroimmunol. 2008;199(1-2):24-34.

24. Groves AK, Barnett SC, Franklin RJ, et al. Repair of demyelinated lesions by transplantation of purified O-2A progenitor cells. Nature. 1993;362(6419):453-455.

25. Richter-Landsberg C, Heinrich M. OLN-93: A new permanent oligodendroglia cell line derived from primary rat brain glial cultures. J Neurosci Res. 1996;45(2):161-173.
26. Prasad R, Giri S, Nath N, Singh I, Singh AK. 5-aminoimidazole-4-carboxamide-1-beta-4-ribofuranoside attenuates experimental autoimmune encephalomyelitis via modulation of endothelial-monocyte interaction. J Neurosci Res. 2006;84(3):614-625.

27. Meares GP, Qin H, Liu Y, Holdbrooks AT, Benveniste EN. AMP-activated protein kinase restricts IFN-gamma signaling. J Immunol. 2013;190(1): 372-380.

28. Mangalam AK, Rattan $\mathrm{R}$, Suhail $\mathrm{H}$, et al. AMP-activated protein kinase suppresses autoimmune central nervous system disease by regulating M1-type macrophage-Th17 axis. J Immunol. 2016;197(3):747-760.

29. Macchi B, Marino-Merlo F, Nocentini U, et al. Role of inflammation and apoptosis in multiple sclerosis: Comparative analysis between the periphery and the central nervous system. J Neuroimmunol. 2015; 287:80-87.

30. Mangano K, Nicoletti A, Patti F, et al. Variable effects of cyclophosphamide in rodent models of experimental allergic encephalomyelitis. Clin Exp Immunol. 2010;159(2):159-168.

31. Bechtold DA, Kapoor R, Smith KJ. Axonal protection using flecainide in experimental autoimmune encephalomyelitis. Ann Neurol. 2004; 55(5):607-616.

32. Tabas I, Ron D. Integrating the mechanisms of apoptosis induced by endoplasmic reticulum stress. Nat Cell Biol. 2011;13(3):184-190.

33. Carrasco DR, Sukhdeo K, Protopopova M, et al. The differentiation and stress response factor XBP-1 drives multiple myeloma pathogenesis. Cancer Cell. 2007;11(4):349-360.

34. Wang M, Kaufman RJ. The impact of the endoplasmic reticulum protein-folding environment on cancer development. Nat Rev Cancer. 2014;14(9):581-597.

35. Hetz C, Mollereau B. Disturbance of endoplasmic reticulum proteostasis in neurodegenerative diseases. Nat Rev Neurosci. 2014;15(4): 233-249.

36. Lin W, Lin Y, Li J, et al. Oligodendrocyte-specific activation of PERK signaling protects mice against experimental autoimmune encephalomyelitis. J Neurosci. 2013;33(14):5980-5991.

37. Paintlia AS, Paintlia MK, Mohan S, Singh AK, Singh I. AMP-activated protein kinase signaling protects oligodendrocytes that restore central nervous system functions in an experimental autoimmune encephalomyelitis model. Am J Pathol. 2013;183(2):526-541.

38. Wang P, Xu TY, Guan YF, et al. Nicotinamide phosphoribosyltransferase protects against ischemic stroke through SIRT1-dependent adenosine monophosphate-activated kinase pathway. Ann Neurol. 2011;69(2):360-374.

39. Mihaylova MM, Shaw RJ. The AMPK signalling pathway coordinates cell growth, autophagy and metabolism. Nat Cell Biol. 2011;13(9): 1016-1023.

40. Nath N, Khan M, Paintlia MK, Singh I, Hoda MN, Giri S. Metformin attenuated the autoimmune disease of the central nervous system in animal models of multiple sclerosis. J Immunol. 2009;182(12): 8005-8014.

41. Singh I, Samuvel DJ, Choi S, Saxena N, Singh AK, Won J. Combination therapy of lovastatin and AMP-activated protein kinase activator improves mitochondrial and peroxisomal functions and clinical disease in experimental autoimmune encephalomyelitis model. Immunology. 2018;154(3):434-451. 